\title{
Risk of new tumors in von Hippel-Lindau patients depends on age and genotype
}

\author{
Marie Louise Mølgaard Binderup, MD' , Esben Budtz-Jørgensen, MSc, PhD ${ }^{1,2}$ and Marie Luise Bisgaard, MD
}

Purpose: The von Hippel-Lindau (vHL) phenotype is variable, which complicates genetic counseling and surveillance. We describe how the rate of new tumor development varies through the lifetimes of vHL patients and how it is influenced by age and genotype.

Methods: In a national cohort study, we included 52 VHL mutation carriers who were retrospectively followed for a total of 799 person-years. From birth to current age, 581 manifestations were diagnosed during 2,583 examinations in the study subjects. Manifestation rates were analyzed using Poisson regression and compared in groups of different ages, tumor sites, and genotypes.

Results: The rate of new tumor development varied significantly with age and was highest at 30-34 years (0.4 new tumors/year). Tumor location further influenced the rate. The risk of retinal tumors was highest in subjects during the teenage years but was highest for cerebellar tumors in subjects during their 30 s. Truncating VHL mutation carriers had a significantly higher manifestation rate compared with missense mutation carriers (hazard ratio $=1.85,95 \%$ confidence interval: $1.06-3.24, P$ value $=0.031)$.

Conclusion: The rate of new manifestation development is not constant throughout the life span of vHL patients; instead, it varies significantly with age and genotype and depends on anatomical location. Retinal surveillance is crucial during the teenage years, whereas cerebellar surveillance is especially important in adulthood.

Genet Med advance online publication 2 April 2015

Key Words: genetic screening/counseling; genotype; manifestation rate; surveillance; von Hippel-Lindau disease

\section{INTRODUCTION}

von Hippel-Lindau disease (vHL) (OMIM number 193300) is an autosomal dominant predisposition to tumor development with an incidence of up to 1 in 36,000 individuals. ${ }^{1}$ It is caused by mutations in the tumor suppressor gene $V H L$ on chromosome 3p25.3.2. The most common vHL manifestations are hemangioblastomas in the central nervous system (CNS) and retina, renal cell carcinomas (RCCs), pheochromocytomas, pancreatic neuroendocrine tumors, and inner-ear endolymphatic sac tumors. ${ }^{2}$ The protein product $\mathrm{pVHL}$ functions in essential cellular pathways such as hypoxia inducible factor (HIF) regulation in the oxygen sensing pathway, assembly of the fibronectin extracellular matrix, cytokine signaling, cell-cycle exit, and various functions that are not yet fully clarified. ${ }^{3}$ Mutations in the VHL gene fall into two main groups: (i) mutations causing a truncated gene product, i.e., frame-shift, nonsense, and splicesite mutations, as well as deletions, and (ii) nontruncating mutations, mainly missense mutations. ${ }^{3}$ Missense mutations are associated with pheochromocytoma development, which in contrast is rare among truncating mutation carriers. ${ }^{3}$

VHL mutation carriers may develop manifestations from early childhood and throughout their lifetime, but there is great variation in relation to when in life the first manifestation develops, which organs are involved, and how severely the individual patient is affected., ${ }^{2,3}$ Previously, focus has been on when and where vHL patients' first manifestations typically occur. ${ }^{1,4-7}$ To date, little is known about how fast or when in life additional new manifestations typically develop once the first manifestation has been diagnosed. Prophylactic surveillance is essential to facilitate early diagnosis of the manifestations and to enable intervention, either surgical or individually intensified follow-up measures of the organ in question. ${ }^{2,8,9}$ The unpredictable mode of disease progression, however, can cause uncertainty for patients and health professionals and complicates both genetic counseling and surveillance organization. Current surveillance guidelines are based on the notion that risk of tumor development is constant throughout a vHL patient's entire lifetime. Apart from age-differentiated initiation of organ-specific surveillance, identical surveillance guidelines are currently applied to all vHL patients and at-risk family members regardless of individual factors. More knowledge about which factors influence manifestation development is essential to improve future surveillance targeting and genetic counseling of $\mathrm{vHL}$ families. We hypothesize that the risk of new vHL manifestations in different organs is not constant throughout life, but, rather, varies with age and depends on individual factors such as the patient's genotype. We aimed to assess how the rate of new vHL manifestation development varies across a typical VHL mutation carrier's life span, to describe age-specific manifestation rates, and to evaluate whether the variation is organ-dependent and if it is influenced by genotype.

${ }^{1}$ Department of Cellular and Molecular Medicine, University of Copenhagen, Copenhagen, Denmark; ${ }^{2}$ Section of Biostatistics, Department of Public Health, University of Copenhagen, Copenhagen, Denmark. Correspondence: Marie Louise Mølgaard Binderup (mlmb@sund.ku.dk)

Submitted 18 October 2014; accepted 17 February 2015; advance online publication 2 April 2015. doi:10.1038/gim.2015.44 


\section{MATERIALS AND METHODS \\ Study cohort}

The study is a retrospective national cohort study. Eligible subjects were living individuals with a molecularly confirmed VHL mutation attending the Danish vHL surveillance program at the time of study inclusion. Subjects were identified in the Danish vHL Database (Danish Data Protection Agency, no. 2009-41-3994) and the Civil Registration System in June 2008. Twenty-six male and 26 female subjects consented to participate, corresponding to almost $90 \%$ (52 of 59) of all known live Danish VHL mutation carriers at the time of study inclusion. Of the remaining seven patients, five were not contacted due to previous privacy requests and two were newly diagnosed and not yet enrolled in surveillance. We collected information about all vHL-related examinations performed and all manifestations diagnosed throughout each subject's lifetime up to the time of study inclusion through detailed interviews and subsequent evaluation of each subject's clinical, radiographic, and histological records. All hospital visits for each patient were evaluated. We included all vHL-related examinations performed as part of the patients' regular surveillance, follow-up examinations if closer surveillance of a particular organ was required, or symptomatic examinations performed due to occurrence of symptoms in between surveillance examinations.

Each vHL manifestation was recorded by type, date, and age at diagnosis. Forty-two subjects had manifestations, whereas 10 were asymptomatic mutation carriers. In total, 581 manifestations were diagnosed during 2,583 examinations, all performed by medical specialists. Almost all of the reported manifestations ( $98 \%$ (571 of 581)) could be verified through medical records; the remaining $2 \%$ were solely reported by the subjects. The majority of the 106 retinal hemangioblastomas were treated by laser photocoagulation at diagnosis and no histological confirmation was possible. Of all other manifestations, not counting retinal hemangioblastomas, 153 had been surgically removed and a histological verification of the diagnosis was obtainable for 92\% (140 of 153). The genetic background of this cohort has been previously described. ${ }^{10}$ Their clinical information has also been previously described. ${ }^{4}$ In brief, Table 1 describes the clinical characteristics of the study cohort. Supplementary Table S1 online shows information about all examinations performed among the subjects. The overall observation time spanned from 1971 to 2008 . The mean observation time was 14.8 years/subject (range: $0.6-37$ years), and overall subjects contributed 799 person-years.

\section{Statistical methods}

Age-dependent manifestation rates including all types of vHL manifestations. To determine how frequent new manifestations were diagnosed at different ages across the patients' life spans, we used Poisson regression to calculate age-dependent manifestation rates in predefined 5-year age intervals from 0 to 60 years. Because surveillance examinations for each manifestation type were initiated at different ages, each subject was included in the analysis from birth to his/her age
Table 1 Characteristics of the 52 included VHL mutation carriers

Median age at study inclusion: 37.5 years (range: 2-64 years)

Number of unrelated families: 21 families (median two subjects/family (range: 1-8))

Median age at diagnosis of first $\mathrm{vHL}$ manifestation: 19.5 years (range: $7-57$ years)

Surveillance attendance ${ }^{a}$

Median number of months each subject has attended a surveillance program: 159 months (range: 7-384 months) Median age at first vHLrelated examination: 19 years (range: $0-57$ years)

Number of manifestations diagnosed

(\% of all manifestations), $N=$ number of subjects with the manifestation type

Retinal hemangioblastomas $106(18.2 \%), N=29$

Cerebellar hemangioblastomas $154(26.2 \%), N=29$

Spinal hemangioblastomas $70(11.9 \%), N=23$

Supratentorial hemangioblastomas $2(0.3 \%), N=2$

Brainstem hemangioblastomas

RCC $8(1.4 \%), N=7$

Renal cysts ${ }^{\mathrm{b}}$ $45(7.7 \%), N=10$

Unspecified renal tumors

$116(19.7 \%), N=25$

Pancreatic cysts ${ }^{b}$

$9(1.5 \%), N=6$

Pancreatic NETS

$41(7.0 \%), N=19$

Unspecified pancreatic tumors

Pheochromocytomas

$1(0.2 \%), N=1$

Paragangliomas

$10(1.7 \%), N=4$

$10(1.7 \%), N=6$

Epididymal cystadenomas

$1(0.2 \%), N=1$

Cysts of the broad uterine ligament

Total

$7(1.2 \%), N=6$

$1(0.2 \%), N=1$

$581(100 \%), N=42$

NET, neuroendocrine tumor; RCC, renal cell carcinoma; vHL, von Hippel-Lindau. aThe ages at which the subjects received $\mathrm{vHL}$ diagnosis and started surveillance varied. Not all subjects were included in a vHL surveillance program from birth as recommended. ${ }^{b}$ Renal and pancreatic cysts were included due to their malignant potential in vHL patients. ${ }^{2}$ When records described "multiple cysts" without further specification, these were counted as a single manifestation.

at study inclusion. All new manifestations diagnosed in each subject's lifetime were counted as an event at the age when it was first diagnosed, regardless of whether it was symptomatic or asymptomatic at diagnosis. In each 5-year age interval, the rate was assumed constant but was allowed to vary across the different age intervals. Subsequently, due to the low number of new manifestations diagnosed in the age intervals younger than 14 and older than 40 years of age, age intervals $0-14$ years and 40-60 years were pooled after successful likelihood ratio testing of this. Robust SEs allowing for heterogeneity in the manifestation rates between the subjects were further calculated in Table 2. These rates are also depicted graphically in Supplementary Figure S1 online.

Comparison of the age-dependent manifestation rates at different tumor sites. To determine whether the rate of 
new manifestation development varied for manifestations at different tumor sites, we chose to calculate age-related manifestation rates separately for cerebellar and retinal hemangioblastomas, because these two manifestations were the most common in our study population and among vHL patients in general. ${ }^{3}$ Each analysis included either only cerebellar or only retinal hemangioblastomas (Table 3 ). Subjects were included with delayed entry from the time of their first CNS imaging or ophthalmoscopy, respectively. All subjects underwent regular surveillance, and from the dates of each examination performed we assessed the age of development of each manifestation, because it must have developed within the time period between the patient's last examination (when the manifestation could not be detected) and the date of the examination at which the manifestation was first diagnosed. It is impossible to determine when manifestations that are diagnosed at a person's first-ever examination have developed, because they can be either newly developed or asymptomatic for many years prior to the first examination. To avoid the potential bias of accumulated asymptomatic manifestations

Table 2 Manifestation rates (new manifestations/year) for all vHL manifestations as a function of age and hazard ratios in relation to the reference interval of 30-34 years

\begin{tabular}{lcccc}
$\begin{array}{l}\text { Age } \\
\text { intervals }\end{array}$ & $\boldsymbol{N}$ & $\begin{array}{c}\text { Manifestation } \\
\text { rates }\end{array}$ & $\begin{array}{c}\text { Hazard ratio } \\
\mathbf{( 9 5 \% ~ C l )}\end{array}$ & $\boldsymbol{P}$ value \\
\hline $0-14$ & 52 & $0.040(0.022-0.071)$ & $0.045(0.024-0.086)$ & $<0.0001$ \\
$15-19$ & 39 & $0.250(0.126-0.497)$ & $0.284(0.122-0.661)$ & 0.004 \\
\hline $20-24$ & 37 & $0.386(0.221-0.676)$ & $0.439(0.225-0.854)$ & 0.015 \\
\hline $25-29$ & 35 & $0.398(0.174-0.911)$ & $0.452(0.181-1.133)$ & 0.090 \\
\hline $30-34$ & 32 & $0.880(0.570-1.360)$ & $1(-)$ & - \\
\hline $35-39$ & 29 & $0.863(0.562-1.326)$ & $0.981(0.570-1.686)$ & 0.944 \\
\hline $40-60$ & 24 & $0.611(0.441-0.846)$ & $0.694(0.423-1.139)$ & 0.148 \\
\hline
\end{tabular}

We included all manifestation types (581 manifestations). Subjects were followed from birth.

$\mathrm{Cl}$, confidence interval; $\mathrm{N}$, number of subjects included in the age interval; $\mathrm{VHL}$, von Hippel-Lindau. at the age of the first examination, we did not include manifestations diagnosed at subjects' first-ever CNS imaging or ophthalmoscopy, respectively.

Comparison of the manifestation rates in different agegroups. In each of the three manifestation rate analyses, (i) all manifestations, (ii) only cerebellar hemangioblastomas, and (iii) only retinal hemangioblastomas, we used hazard ratios to compare the manifestation rate in each age group to the group with the highest rate in each analysis as the reference group (Tables 2 and 3 ).

Illustration of life-span variation of the manifestation rates. Additionally, to better illustrate how the patterns of the manifestation rates vary with age across patients' life spans, we further used a restricted cubic spline function to produce smooth graphs (Figures 1 and 2 and Supplementary Figure S1 online). In these functions, none of the 5-year age groups were pooled. We chose the smooth graphs instead of the curves in which the rates are held constant in each age interval, because the smooth rate across age intervals better supports the presumed gradual natural development of vHL tumors. The graphs with constant rates in each 5-year interval including cerebellar and retinal hemangioblastomas only are shown in the Supplementary Figures S2 and S3 online, respectively.

Effect of genotype on manifestation rates. To determine the effect of VHL mutation type on manifestation development, subjects were divided into two genotype groups: (i) carriers of missense mutations defined as a single nucleotide substitution leading to substitution of an amino acid and (ii) carriers of truncating mutations defined as mutations predicted to truncate the gene product (nonsense, frame-shift, and splice-site mutations, along with exon deletions). We used two models to assess whether the genotype affects the manifestation rate. In the first model, we assumed that a genotype effect was constant at all ages, i.e., that the ratio between manifestation rates in the two

Table 3. Manifestation rates (new manifestations/year) of cerebellar and retinal hemangioblastomas as a function of age and hazard ratios in relation to the age intervals with the highest manifestation rates

\begin{tabular}{|c|c|c|c|c|c|c|c|c|}
\hline \multirow[b]{2}{*}{$\begin{array}{l}\text { Age } \\
\text { intervals }\end{array}$} & \multicolumn{4}{|c|}{ Cerebellar hemangioblastomas } & \multicolumn{4}{|c|}{ Retinal hemangioblastomas } \\
\hline & $N$ & $\begin{array}{c}\text { Manifestation rate } \\
(95 \% \mathrm{Cl})\end{array}$ & $\begin{array}{l}\text { Hazard ratio } \\
(95 \% \mathrm{Cl})\end{array}$ & $P$ value & $N$ & $\begin{array}{c}\text { Manifestation rate } \\
(95 \% \mathrm{Cl})\end{array}$ & $\begin{array}{l}\text { Hazard ratio }^{b} \\
(95 \% \mathrm{Cl})\end{array}$ & $\begin{array}{c}P \\
\text { value }\end{array}$ \\
\hline $0-14$ & 13 & $0.066(0.023-0.192)$ & $0.116(0.035-0.388)$ & 0.0005 & 20 & $0.080(0.033-0.194)$ & $0.156(0.041-0.601)$ & 0.0069 \\
\hline $15-19$ & 10 & $0.117(0.035-0.393)$ & $0.205(0.068-0.616)$ & 0.0048 & 11 & $0.513(0.219-1.204)$ & $1(-)$ & - \\
\hline $20-24$ & 19 & $0.202(0.086-0.476)$ & $0.353(0.131-0.952)$ & 0.0397 & 19 & $0.307(0.126-0.747)$ & $0.599(0.193-1.854)$ & 0.3739 \\
\hline $25-29$ & 21 & $0.107(0.044-0.259)$ & $0.187(0.065-0.539)$ & 0.0019 & 22 & $0.032(0.008-0.135)$ & $0.062(0.015-0.261)$ & 0.0001 \\
\hline $30-34$ & 25 & $0.351(0.200-0.615)$ & $0.614(0.282-1.338)$ & 0.2197 & 25 & $0.101(0.034-0.298)$ & $0.196(0.053-0.735)$ & 0.0156 \\
\hline $35-39$ & 26 & $0.572(0.329-0.993)$ & $1(-)$ & - & 27 & $0.099(0.038-0.257)$ & $0.193(0.054-0.696)$ & 0.0119 \\
\hline $40-60$ & 22 & $0.351(0.236-0.521)$ & $0.614(0.306-1.232)$ & 0.1696 & 22 & $0.092(0.049-0.172)$ & $0.176(0.058-0.552)$ & 0.0028 \\
\hline
\end{tabular}

We included only cerebellar and retinal hemangioblastomas. Subjects were included with delayed entry from the time of their first central nervous system imaging or ophthalmoscopy, respectively.

$\mathrm{Cl}$, confidence interval; $N$, number of subjects included in the age interval.

${ }^{a}$ Hazard ratios in relation to the reference interval of 35-39 years. ${ }^{b}$ Hazard ratios in relation to the reference interval of 15-19 years. 

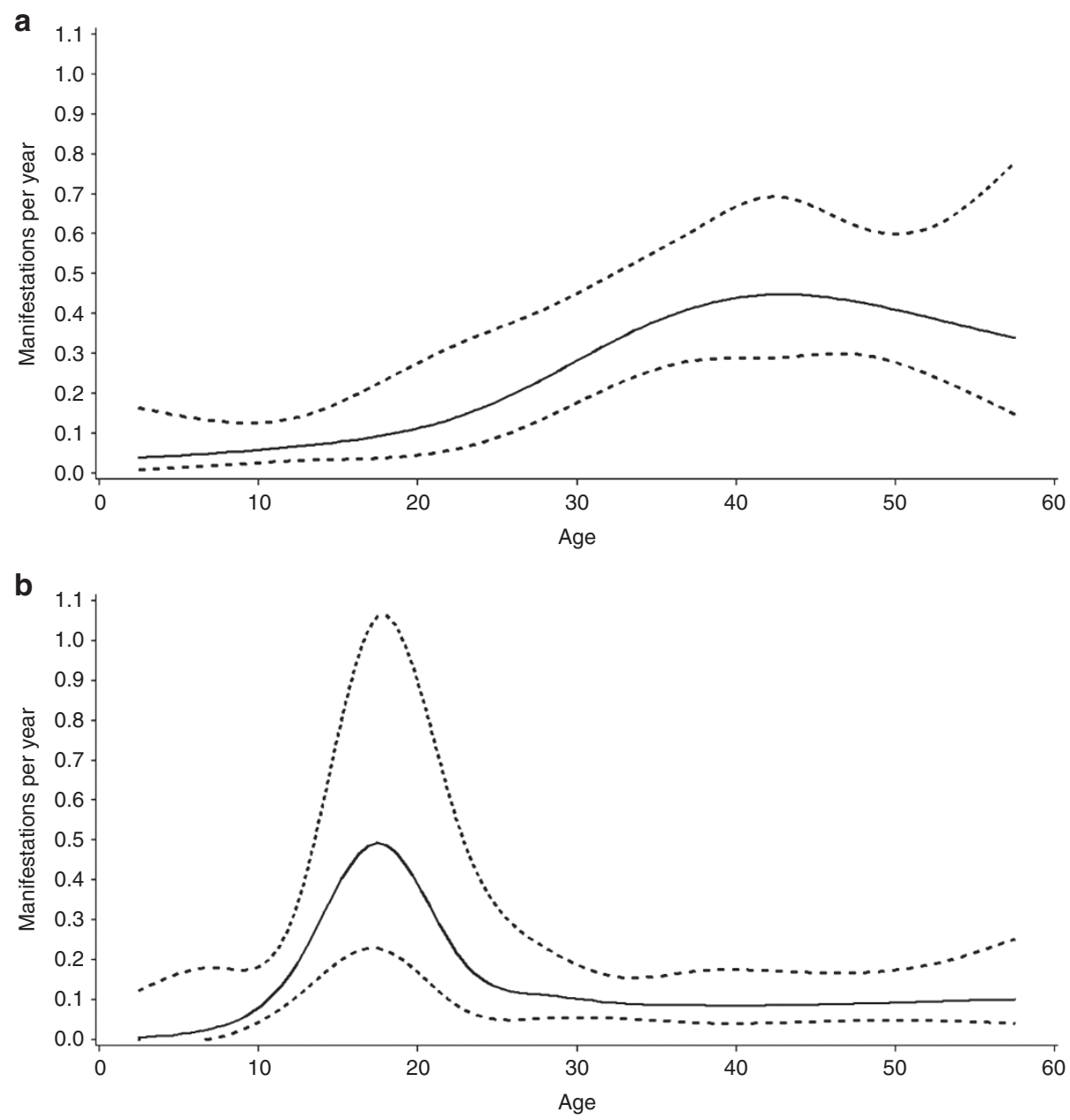

Figure 1 vHL manifestation rate as a function of age. (a) New cerebellar hemangioblastomas. (b) New retinal hemangioblastomas. Continuous line: the number of new manifestations diagnosed per year as a function of age. Dotted line: $95 \%$ confidence interval. Poisson regression was used to calculate age-dependent manifestation rates in predefined 5 -year age intervals. A restricted cubic spline function was used to depict the rates in smooth graphs. Subjects were included with delayed entry from the time of their first magnetic resonance imaging (MRI)/computed tomography (CT) of the central nervous system (a)/ophthalmoscopy (b). Figure $1 \mathbf{a}$ is based on 120 cerebellar hemangioblastomas. Figure $1 \mathbf{b}$ is based on 88 retinal hemangioblastomas.

genotype groups was the same in all age intervals. We therefore included the genotype variable along with the age covariate in the Poisson regression model with all manifestations. We then calculated this joint hazard ratio and its $95 \%$ confidence interval (CI). In the second model, we explored whether the difference in manifestation rates between the two genotype groups was actually related to age by also including an interaction term between age and genotype in the model and then comparing the profiles of the two genotype groups. Finally, we tested the hypothesis of a constant genotype effect by comparing models one and two using a likelihood ratio test. Figure 2 shows these age-dependent manifestation rate profiles in the two genotype groups (the second model) using smooth curves. Supplementary Figures S4 and S5 online depict the first and second model, respectively, using constant rates. Additionally, we explored the genotype effect of manifestation rates when only cerebellar hemangioblastomas or retinal hemangioblastomas were considered (Figure 2).

Statistical analysis was performed using SAS version 9.3 (SAS Institute, Cary, NC).
The study was approved by the Danish Regional Committees on Biomedical Research Ethics (H-2-2010-012) and the Data Protection Agency (2009-41-3994). All included subjects gave their written informed consent to participation.

\section{RESULTS}

Overall, 581 manifestations were diagnosed throughout the life spans of the 52 VHL mutation carriers included. When considering all vHL manifestations diagnosed, new manifestations were most frequently diagnosed in patients in their 30s, corresponding to almost one new manifestation per year (Table 2, Supplementary Figure S1 online). The rate of new manifestations diagnosed varied markedly over the course of the patients' lives, initially corresponding to 0.04 new manifestation per year until their teenage years; thereafter, it increased with age and peaked at the age interval of 30-34 years (Supplementary Figure S1 online). The manifestation rates of the younger age groups were significantly lower than at the reference interval of 30-34 years (Table 2). 

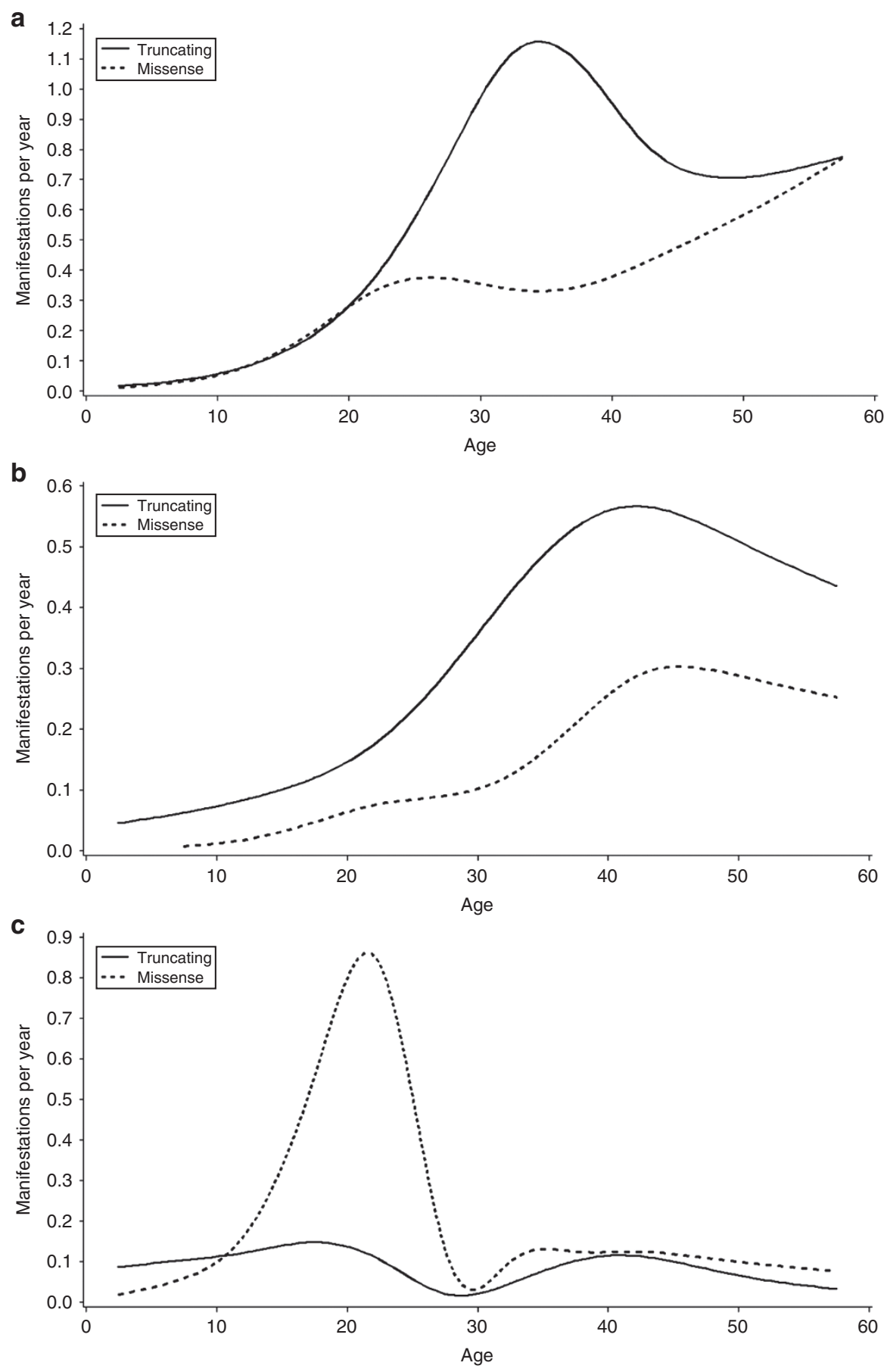

Figure 2 Effect of genotype on age-dependent manifestation rates for (a) all vHL manifestations, (b) only cerebellar hemangioblastomas, and (c) only retinal hemangioblastomas. Poisson regression was used to calculate manifestation rates (the number of new manifestations diagnosed per year) in predefined 5-year age intervals. A restricted cubic spline function was used to depict the rates in smooth graphs. (a) Includes all vHL manifestation types (581 manifestations). Because surveillance examinations for the different manifestation types were initiated at different ages, each subject was included in the analysis from birth. (b) Includes only cerebellar hemangioblastomas (120 tumors) and (c) includes only retinal hemangioblastomas (88 tumors). In the analyses for $(\mathbf{b})$ and $(\mathbf{c})$, subjects were included with delayed entry from the time of their first central nervous system (CNS) imaging or ophthalmoscopy, respectively. Also, any cerebellar or retinal tumors diagnosed at either the first CNS imaging or ophthalmoscopy, respectively, were excluded. Continuous line: age-dependent manifestation rate based on new manifestations diagnosed in patients with truncating germline VHL mutations. Dotted line: manifestation rate based on new manifestations diagnosed in patients with missense germline mutations See Supplementary Table S2 online for a full list of the included subjects' genotypes. 
When we calculated age-related manifestations rates for cerebellar hemangioblastomas only, new lesions were diagnosed at the highest rate in subjects in their 30s, roughly corresponding to a diagnosis of approximately 0.5 new hemangioblastoma per year (Table 3, Figure 1a). Conversely, the rate corresponded to less than 0.1 new hemangioblastoma per year until the subjects were in their 20s, and was significantly lower at ages under 30 when compared with the reference interval of 35-39 years.

When only retinal hemangioblastomas were included in the calculation of the age-related manifestation rate, the highest rate of new lesion diagnosis occurred between 15 and 19 years, corresponding to a diagnosis of approximately 0.5 new hemangioblastoma per year (Table 3, Figure 1b). At both younger and older ages, the rates were significantly lower compared with the reference interval of 15-19 years. This rate stabilized from the subjects' 30 s at a level of less than 0.1 new manifestation diagnosed per year.

Thirty subjects had a truncating mutation, whereas $22 \mathrm{had}$ a missense mutation. When all manifestations were included and we assumed the same genotype effect in all age groups, truncating mutation carriers had a significantly higher overall manifestation rate compared with missense mutation carriers (hazard ratio $=1.85,95 \%$ CI: $1.05-3.25, P$ value $=0.033)$. When we included a genotype-age interaction, and thereby allowed the age-dependent manifestation rate to vary freely in the two genotype groups, the profiles were similar until the second decade of life; thereafter, the rate for truncating mutation carriers increased markedly compared with those of missense mutation carriers (Figure 2). However, when the model allowing for an age-genotype interaction was compared with the model without interaction (Supplementary Figures S4 and S5 online), they were not significantly different $(P$ value $=0.42)$, meaning that the hypothesis of a constant effect of genotype throughout life that does not depend on age could not be rejected.

When including only cerebellar hemangioblastomas, truncating mutation carriers had double the risk of developing a new manifestation (hazard ratio $=2.259,95 \%$ CI: 1.005-5.077, $P$ value $=0.049)$, but they had an overall lower risk of retinal hemangioblastomas (hazard ratio $=0.391,95 \%$ CI: $0.211-$ $0.727, P$ value $=0.003)$ compared with missense mutation carriers. The rates of variation with age are depicted using smooth graphs in (Figure 2a,b).

\section{DISCUSSION}

The present study is the first to demonstrate that the number of new vHL manifestations diagnosed per year is not constant throughout the life span of a typical VHL mutation carrier, but actually varies significantly with age. It is also the first to describe how this pattern of manifestation development varies with genotype and depends on the anatomical location of the lesions. Further, our results provide the basis for estimating the probability of developing new vHL manifestations at various ages. Previous studies of vHL manifestation development have predominantly focused on either total or cumulative manifestation frequencies or on growth patterns of individual tumors. ${ }^{6,711-15}$ The few studies that have assessed growth patterns of vHL tumors have mostly assessed growth rates of individual tumors in relation to observation time regardless of the patients' ages. ${ }^{12,13}$ Furthermore, many of these studies have solely included patients with clinical vHL or a specific manifestation type and have not taken asymptomatic VHL mutations carriers into account, ${ }^{6,12-14}$ which may bias the frequency estimates. This study uniquely includes a longitudinal life-span perspective in relation to manifestation development. No other study has previously included data from all examinations performed during each patient's entire life span or described how the natural occurrence of new manifestations progresses with age. Lonser et al. ${ }^{14}$ recently investigated the natural history of CNS hemangioblastoma development among vHL patients in a large prospective cohort. In contrast with our results, they found that patients between 12 and 20 years of age developed significantly more new tumors per year compared with older subjects. However, the results of the study by Lonser et al. ${ }^{14}$ are not directly comparable with our findings because they exclusively included symptomatic patients who had already had at least one CNS hemangioblastoma diagnosed. Therefore, their results cannot necessarily be applied to all $V H L$ mutation carriers. Because we included almost all known Danish VHL mutation carriers regardless of phenotype, our study population is assessed to be representative for vHL patients in general. It should, however, be noted that any study population selected based on a known diagnosis may overestimate the phenotype of a disease because it will not include any patients/families with mild phenotypes who have not been diagnosed or even misdiagnosed.

Owing to the retrospective study design, new manifestations were evaluated through medical records. The unique Danish national health registers on which the study is based have allowed us to include a substantial, well-documented, and almost complete material covering each patient's entire life span. It has previously been suggested that vHL surveillance might have been insufficient before guideline establishment in approximately $1990,{ }^{16}$ which could theoretically lead to an underestimation of new manifestations in the earlier time periods. However, according to our previous study of the same population, surveillance frequencies before 1993 were, on average, remarkably, consistent with present recommendations, although wider ranges were seen. ${ }^{4}$ Based on this, we assess that the included material provides a complete and accurate manifestation count in the entire observation period.

\section{Variation in manifestation rate depends on the anatomical location of the tumor}

We found a marked difference in how the manifestation rate varied with age for hemangioblastomas in the cerebellum and those in the retina. Consistent with our results, Lonser et al. ${ }^{14}$ recently found that established CNS hemangioblastomas grew at significantly different rates depending on their anatomical location in the CNS. The contrasting manifestation rate profiles found in this study point toward two distinct development 
patterns at the two anatomical locations, although tumors in both locations are identical in their histopathology and origin. ${ }^{2}$ Several studies have indicated that VHL deficient hemangioblastoma precursor cells, tumorlets, are established during embryonic development, although the exact tumorigenesis is still unknown. ${ }^{17-22}$ Thus, from birth, vHL patients may harbor numerous microscopic tumorlets in the CNS, including the retina, and similar growth patterns would be expected. Differing anatomical and diagnostic limitations may account for some of the observed disparity in tumor development at the two sites. Consistent with the present findings, a slighter growth in the anatomically restricted retina would result in detectable signs or symptoms compared with in the cerebellum, where longer asymptomatic growth periods are possible. The initiation and/ or promotion of tumor growth are likely affected by local factors acting independently at separate locations. Although no specific factors have been identified, several possible influences on vHL tumorigenesis have been suggested, including local micro-environmental stimuli and additional genetic alterations. ${ }^{13,17}$

In our analysis of manifestation rates for cerebellar or retinal hemangioblastomas, subjects were included with delayed entry from the time of their first examination of the organ in question. Also, all the manifestations diagnosed at either the first CNS imaging or ophthalmoscopy, respectively, were excluded. This was done to eliminate the potential bias of overestimating manifestation count at the first examinations due to accumulated asymptomatic manifestations that could have developed many years prior to the first examination. All manifestations diagnosed at subsequent examinations will have developed from the time of the last examination to the time of diagnosis, allowing for an accurate estimate of the patients' ages at the time of each manifestation's development. Because we exclude the manifestations diagnosed at the first examination, the true manifestation rates in the younger age groups when surveillance is initiated may be even higher than demonstrated. The approach with delayed entry could not be applied in the analysis including all manifestation types, because surveillance for each type was initiated at different ages. Thus, in that analysis the manifestation rates in the younger age groups may, by contrast, be overestimated because we risk the bias of a falsely high manifestation development burden at the time of a patient's first examination.

\section{Genotype influences rate of new tumor development in adulthood}

The present study demonstrates that missense mutation carriers have a significantly lower overall new manifestation rate compared with truncating mutation carriers. Further, this difference for the two genotype groups may be age-related, so that the manifestation rate profiles for the two genotype groups particularly differ from the patients' 20s. Most germline missense mutations produce an altered pVHL that might have residual functionality, whereas truncating mutations generally lead to a lack of functional pVHL or possibly complete gene product loss due to nonsense mediated RNA decay. ${ }^{23,24}$ Even though missense mutation carriers are known to have a higher pheochromocytoma risk, their overall milder phenotype is most likely due to a resulting $\mathrm{pVHL}$ that is defective in fibronectin matrix assembly, associated with pheochromocytoma development, ${ }^{25}$ but retains some function in the HIF pathway. Because faulty HIF regulation is a key step in RCC and hemangioblastoma development, ${ }^{24,26-28}$ missense mutation carriers are likely to have a slighter, possibly age-delayed, rate of new hemangioblastoma and RCC development. The higher adulthood manifestation rate in truncating mutation carriers seems to result from higher rates of new CNS lesions. For cerebellar hemangioblastomas, the overall manifestation rate for truncating mutation carriers was significantly higher than for missense mutation carriers. By contrast, the overall rate of retinal hemangioblastomas was significantly lower in truncating mutation carriers compared with missense mutation carriers. The milder retinal phenotype in truncating mutation carriers could, in part, be due to simultaneous loss of the $V H L$-adjacent $B R K 1$ gene in patients with $V H L$ exon-one deletions, which is associated with a significantly lower retinal hemangioblastoma risk. ${ }^{29,30}$ However, other unknown mechanisms must also affect retinal hemangioblastoma development, because simultaneous $B R K 1$ deletions were identified in only three of our eight subjects with VHL exon-one deletions.

The biological consequences of $V H L$ mutations are likely to also depend on the total amount of functional protein product remaining in the cell, and not only on the cellular pathways affected by a germline mutation. In line with Knudson's two-hit hypothesis, tumor development requires both an inherited VHL germline mutation in one allele (first hit), present in all the patient's cells, and a somatic loss or inactivation of the wild-type allele (second hit). ${ }^{31}$ However, studies of second hit in vHL lesions have so far been based on only a small number of tumors, ${ }^{32-36}$ and further studies are needed to elucidate the role the second hit in tumor development.

We broadly distinguished genotype into two main groups according to which mutations were assessed to have similar biological consequences. Our material was not large enough to make further distinctions, although some biological variety may exist in genotype subgroups, possibly influencing manifestation rates. It should be mentioned that the two largest included families each comprises eight individuals and each family belongs to a different genotype group. However, we did not assess that the overrepresentation of subjects with the same genotype biased the results because their phenotypes vary considerably within each family, as do the phenotypes of the other included families.

\section{Surveillance recommendations}

Because of the unpredictable phenotypic expression of vHL, it is difficult to find the balance between efficient surveillance recommendations with minimal frequencies of missed life-threatening or disabling manifestations as well as minimal psychological strain on the families. Two studies have systematically investigated vHL surveillance recommendations in 
relation to optimal surveillance initiation and frequency., ${ }^{4,9}$ So far, individualized surveillance in relation to factors such as age or genotype has not been explored.

Our results show that regular retinal screening is especially important in vHL patients during their teenage years, when the rate of new manifestations diagnosed is highest. In line with the current surveillance guidelines, we recommend that annual ophthalmoscopy should start in early childhood. ${ }^{8}$ This further corresponds with previous findings of annual examinations as the most optimal retinal surveillance frequency. ${ }^{4}$ By contrast, Kurizinga et al. ${ }^{9}$ recently recommended that retinal surveillance should be performed every second year instead of yearly as currently recommended. However, both previous studies are based on overall manifestation frequencies that did not take into account that the risk of a new retinal manifestation may differ for children, teenagers, and adults. Based on this study, retinal surveillance could possibly be relaxed later in life, because the manifestation rates seem to decrease to less than one lesion every 10 years. Further knowledge is needed about the individual risk factors of the patients who do develop new retinal tumors in adulthood before such recommendations can be applied to clinical practice.

The CNS surveillance should be given particular attention once the patients are in their 30s because new tumors develop significantly faster than they do at younger ages. We recommend CNS surveillance every 1-2 years, starting from the late teens, in compliance with current guidelines as well as the two previous surveillance studies. ${ }^{4,8,9}$ We stress that surveillance recommendations apply to asymptomatic patients without manifestations in the concerned organ system. Once a manifestation is diagnosed, individual follow-up should be assessed.

We have demonstrated that manifestation development rates in adults are highly related to genotype and provide a great potential for future surveillance targeting. However, due to our limited study population, only a crude division into two genotype groups was possible, and these results alone cannot form the basis of specific surveillance recommendations. Longitudinal studies mapping genotypic agerelated manifestation rates in a more differential manner are necessary.

This study provides the initial basis for a targeted clinical assessment of lifelong vHL tumor development that is useful in surveillance planning as well as in genetic counseling.

\section{SUPPLEMENTARY MATERIAL}

Supplementary material is linked to the online version of the paper at http://www.nature.com/gim

\section{ACKNOWLEDGMENTS}

We thank all participating vHL patients, their families, and the Danish vHL patient association for their help. The study was supported financially by the Danish Cancer Society, the Lundbeck Foundation, and the Michaelsen Foundation. The study sponsors only provided financial support and had no role in the study design; the collection, analysis, and interpretation of data; the writing of the report; or the decision to submit the report for publication.

\section{DISCLOSURE}

The authors declare no conflict of interest.

\section{REFERENCES}

1. Maher ER, Iselius L, Yates JR, et al. Von Hippel-Lindau disease: a genetic study. J Med Genet 1991;28:443-447.

2. Maher ER, Neumann HP, Richard S. von Hippel-Lindau disease: a clinical and scientific review. Eur J Hum Genet 2011;19:617-623.

3. Nordstrom-O'Brien M, van der Luijt RB, van Rooijen E, et al. Genetic analysis of von Hippel-Lindau disease. Hum Mutat 2010;31:521-537.

4. Poulsen ML, Budtz-Jørgensen E, Bisgaard ML. Surveillance in von Hippel-Lindau disease (vHL). Clin Genet 2010;77:49-59.

5. Maddock IR, Moran A, Maher ER, et al. A genetic register for von Hippel-Lindau disease. J Med Genet 1996;33:120-127.

6. Maher ER, Yates JR, Harries R, et al. Clinical features and natural history of von Hippel-Lindau disease. Q J Med 1990;77:1151-1163.

7. Bender BU, Eng C, Olschewski M, et al. VHL C.505 T>C mutation confers a high age related penetrance but no increased overall mortality. J Med Genet 2001;38:508-514.

8. Binderup ML, Bisgaard ML, Harbud V, et al.; Danish vHL Coordination Group. Von Hippel-Lindau disease ( $\mathrm{VHL}$ ). National clinical guideline for diagnosis and surveillance in Denmark. 3rd edition. Dan Med J 2013;60:B4763.

9. Kruizinga RC, Sluiter WJ, de Vries EG, et al. Calculating optimal surveillance for detection of von Hippel-Lindau-related manifestations. Endocr Relat Cancer 2014:21:63-71.

10. Dandanell M, Friis-Hansen L, Sunde L, Nielsen FC, Hansen TV. Identification of 3 novel VHL germ-line mutations in Danish VHL patients. BMC Med Genet 2012;13:54.

11. Conway JE, Chou D, Clatterbuck RE, Brem H, Long DM, Rigamonti D. Hemangioblastomas of the central nervous system in von Hippel-Lindau syndrome and sporadic disease. Neurosurgery 2001;48:55-62; discussion 62.

12. Ammerman JM, Lonser RR, Dambrosia J, Butman JA, Oldfield EH. Long-term natural history of hemangioblastomas in patients with von Hippel-Lindau disease: implications for treatment. J Neurosurg 2006;105:248-255.

13. Wanebo JE, Lonser RR, Glenn GM, Oldfield EH. The natural history of hemangioblastomas of the central nervous system in patients with von HippelLindau disease. J Neurosurg 2003;98:82-94.

14. Lonser RR, Butman JA, Huntoon K, et al. Prospective natural history study of central nervous system hemangioblastomas in von Hippel-Lindau disease. J Neurosurg 2014;120:1055-1062.

15. Zhang J, Pan JH, Dong BJ, Xue W, Liu DM, Huang YR. Active surveillance of renal masses in von Hippel-Lindau disease: growth rates and clinical outcome over a median follow-up period of 56 months. Fam Cancer 2012;11:209-214.

16. Lalloo F, Clancy T, Huson SM, et al. Life expectancy in hereditary cancer predisposing diseases: an observational study. J Med Genet 2012;49:264-269.

17. Park DM, Zhuang Z, Chen L, et al. von Hippel-Lindau disease-associated hemangioblastomas are derived from embryologic multipotent cells. PLoS Med 2007;4:e60.

18. Vortmeyer AO, Frank S, Jeong SY, et al. Developmental arrest of angioblastic lineage initiates tumorigenesis in von Hippel-Lindau disease. Cancer Res 2003;63:7051-7055

19. Vortmeyer AO, Falke EA, Gläsker S, Li J, Oldfield EH. Nervous system involvement in von Hippel-Lindau disease: pathology and mechanisms. Acta Neuropathol 2013;125:333-350.

20. Chan CC, Chew EY, Shen D, Hackett J, Zhuang Z. Expression of stem cells markers in ocular hemangioblastoma associated with von Hippel-Lindau (VHL) disease. Mol Vis 2005;11:697-704.

21. Vortmeyer $A O$, Tran MG, Zeng W, et al. Evolution of VHL tumourigenesis in nerve root tissue. J Pathol 2006;210:374-382.

22. Zhuang Z, Frerich JM, Huntoon $K$, et al. Tumor derived vasculogenesis in von Hippel-Lindau disease-associated tumors. Sci Rep 2014;4:4102.

23. Micale L, Muscarella LA, Marzulli M, et al. VHL frameshift mutation as target of nonsense-mediated mRNA decay in Drosophila melanogaster and human HEK293 cell line. J Biomed Biotechno/ 2009;2009:860761. 
24. Ong KR, Woodward ER, Killick P, Lim C, Macdonald F, Maher ER. Genotypephenotype correlations in von Hippel-Lindau disease. Hum Mutat 2007;28: 143-149.

25. Hoffman MA, Ohh M, Yang H, KIco JM, Ivan M, Kaelin WG Jr. von HippelLindau protein mutants linked to type $2 \mathrm{C} \mathrm{VHL}$ disease preserve the ability to downregulate HIF. Hum Mol Genet 2001;10:1019-1027.

26. Clifford SC, Cockman ME, Smallwood AC, et al. Contrasting effects on HIF1alpha regulation by disease-causing $\mathrm{PVHL}$ mutations correlate with patterns of tumourigenesis in von Hippel-Lindau disease. Hum Mol Genet 2001;10: 1029-1038.

27. Gallou C, Chauveau D, Richard S, et al. Genotype-phenotype correlation in von Hippel-Lindau families with renal lesions. Hum Mutat 2004;24:215-224.

28. Gläsker S, Bender BU, Apel TW, et al. The impact of molecular genetic analysis of the VHL gene in patients with haemangioblastomas of the central nervous system. J Neurol Neurosurg Psychiatry 1999;67:758-762.

29. Franke $G$, Bausch B, Hoffmann MM, et al. Alu-Alu recombination underlies the vast majority of large $\mathrm{VHL}$ germline deletions: Molecular characterization and genotype-phenotype correlations in VHL patients. Hum Mutat 2009;30:776-786.
30. McNeill A, Rattenberry E, Barber R, Killick P, MacDonald F, Maher ER. Genotype-phenotype correlations in VHL exon deletions. Am J Med Genet A 2009;149A:2147-2151.

31. Knudson AG Jr. Mutation and cancer: statistical study of retinoblastoma. Proc Natl Acad Sci USA 1971;68:820-823.

32. Gläsker S, Bender BU, Apel TW, et al. Reconsideration of biallelic inactivation of the $\mathrm{VHL}$ tumour suppressor gene in hemangioblastomas of the central nervous system. J Neurol Neurosurg Psychiatry 2001;70:644-648.

33. Gläsker S, Sohn TS, Okamoto H, et al. Second hit deletion size in von HippelLindau disease. Ann Neurol 2006;59:105-110.

34. Prowse AH, Webster AR, Richards FM, et al. Somatic inactivation of the VHL gene in Von Hippel-Lindau disease tumors. Am J Hum Genet 1997;60: 765-771.

35. Wait SD, Vortmeyer AO, Lonser RR, et al. Somatic mutations in VHL germline deletion kindred correlate with mild phenotype. Ann Neurol 2004;55:236-240.

36. Vortmeyer AO, Huang SC, Pack SD, et al. Somatic point mutation of the wild-type allele detected in tumors of patients with VHL germline deletion. Oncogene 2002;21:1167-1170. 